\title{
La inteligencia emocional en la educación, tips para desarrollar en el aula
}

\section{Emotional intelligence in education, tips for classroom development}

\author{
DOI: $10.46932 / \mathrm{sfjdv} 3 \mathrm{n} 1-122$
}

Received in: January $20^{\text {th }}, 2022$

Accepted in: February $1^{\text {st }}, 2022$

\author{
Mayté Cadena González \\ Maestra en Educación Superior \\ Instituicion: Universidad Autónoma de Campeche \\ Direnccion: Avenida Agustín Melgar s/n Col. Buenavista CP. 24039, San Francisco de Campeche \\ México \\ Correio eletrónico: macadena@uacam.mx \\ María Alejandra Sarmiento Bojórquez \\ Doctora en Educación \\ Instituicion: Universidad Autónoma de Campeche \\ Direnccion: Avenida Agustín Melgar s/n Col. Buenavista CP. 24039 San Francisco de Campeche \\ México \\ Correio eletrónico: masarmie@uacam.mx

\section{Juan Fernando Casanova Rosado} \\ Doctor en Educación \\ Instituicion: Universidad Autónoma de Campeche \\ Direnccion: Avenida Agustín Melgar s/n Col. Buenavista CP. 24039 San Francisco de Campeche \\ México \\ Correio eletrónico: jfcasano@uacam.mx
}

\section{RESUMEN}

Hoy en día las TIC han ido innovando la forma en que el alumno aprende, ya que se considera que el alumno debe: aprender a conocer, aprender a hacer, aprender a vivir juntos y aprender a ser. Generalmente los docentes cubrimos perfectamente bien las dos primeras premisas y quizás la tercera, pero no atendemos la última ¿estamos contribuyendo a su formación integral?; ¿realmente estamos propiciando el desarrollo de competencias? Necesitamos aplicar estrategias o ciertos tips para propiciar en él la aptitud tanto personal como social, para que alcance éxito en la vida. Necesitamos que se conozcan ellos mismos para canalizar sus emociones a favor de su aprendizaje. En este trabajo basado en la experiencia se proponen tips que el docente debe utilizar para hacer consiente al alumno de sus emociones y pueda desarrollar su Inteligencia Emocional y esta a su vez impacte en su proceso de aprendizaje ayudando al desarrollo de competencias. La inteligencia emocional implica, entre otras habilidades, ser capaz de motivarse y persistir frente a las decepciones; para desarrollar la inteligencia emocional el alumno debe: expresar sus emociones, decir lo que piense, aplicar el esquema de valores, reconocer aciertos y desaciertos, leer libros de superación personal, adoptar una actitud de... yo puedo, trabajar en equipo para fomentar cooperación. Queremos que el alumno no solamente tenga éxito académicamente, sino también éxito en su vida.

Palabras clave: aprendizaje, competencias, inteligencia emocional. 


\begin{abstract}
Today the ICT has been innovating the way the student learns, since it is considered that the student must: learn to know, learn to do, learn to live together, and learn to be. Generally, the teachers cover perfectly the first two premises and perhaps the third, but we do not take care of the last one. Are we contributing to their holistic formation? Are we really encouraging the development of competences? We need to apply strategies or certain tips to promote personal and social fitness to achieve success in life. We need them to know themselves to channel their emotions in favor of their learning. In this work based on experience are proposed tips that the teacher should use to make the student aware of their emotions and can develop their Emotional Intelligence and this in turn impact on their learning process helping the development of skills. Emotional intelligence involves, among other skills, being able to motivate and persist in the face of disappointments; To develop emotional intelligence the student must: express their emotions, say what they think, apply the scheme of values, recognize successes and failures, read books of personal improvement, adopt an attitude of ... I can, work as a team to foster cooperation. We want the student not only to succeed academically, but also to succeed in his or her life.
\end{abstract}

Keywords: learning, emotional intelligence, competences.

\title{
1 INTRODUCCIÓN
}

En la actualidad seguimos en la búsqueda de poder educar de manera integral a los jóvenes que serán los futuros profesionista que guíen el destino de las naciones. Pero como docentes ¿estamos haciendo lo necesario para lograrlo? Enseñamos a nuestros alumnos el conocimiento científico de las cosas y queremos que aprendan los contenidos plasmados en los programas de estudios, luego medimos estos conocimientos con pruebas estandarizadas para comprobarlo. ¿Realmente desarrollan las competencias necesarias para su vida académica y social? ¿Y la parte emocional?, son tantas preguntas que nos podemos hacer, sin embargo, hay que entender que:

El siglo XXI nos ha traído una nueva forma de ver la realidad más diversa sobre el funcionamiento de las personas y estamos tomando conciencia de forma lenta, aunque progresiva, de la necesidad de que la educación de los aspectos emocionales y sociales sean atendidos y apoyados por la familia, pero también de forma explícita por la escuela y la sociedad" (Fernández-Berrocal \& Ruiz Aranda, 2008, p. 425)

Para esta nueva educación existen muchas tendencias sobre el método que debemos utilizar. México basa su educación en un modelo por competencias en el cual destaca una educación Integral para que los jóvenes aprendan no solo conocimientos científicos sino también valores.

La Educación Media Superior (EMS) es a la cual se pone especial énfasis, ya que es donde se forman a los individuos que tienen edad de ejercer sus derechos y obligaciones como ciudadanos. Es en esta etapa de su vida donde desarrolla una serie de actitudes y valores que tendrán en un futuro un impacto positivo o negativo en su comunidad, es aquí donde sus emociones juegan un papel importante para la decisión del rumbo que llevará su vida. Para ello las autoridades han instituido diversos programas como 
apoyo para que las escuelas puedan educar aspectos emocionales; uno de ellos es el programa Construye T, el cual es dirigido y financiado por la Secretaría de Educación Pública (SEP), a través de la Subsecretaría de Educación Media Superior. El objetivo de este programa es fortalecer las capacidades de la escuela para desarrollar habilidades socioemocionales en los jóvenes, y así mejorar el ambiente escolar (SEP, 2015). El programa está bien estructurado, pero no siempre se cuenta con la preparación y el tiempo suficiente para el desarrollo de todas las actividades. Nuestra experiencia docente nos ha llevado estudiar y buscar la mejor alternativa para implementar ciertos Tips durante nuestras clases en el aula y poder ayudar a nuestros alumnos a conocer sus emociones y poder desarrollar capacidades que los ayuden en su formación integral.

Los docentes tienen la responsabilidad de emplear el método adecuado para hacer que el alumno cumpla con el proceso enseñanza-aprendizaje, nos ha tocado vivir en una era tecnológica donde la información fluye a gran velocidad, y en ciertas ocasiones aturde, unido a los cambios emocionales que presentan en la adolescencia se tiene que buscar propiciar en los jóvenes una actitud positiva y encontrar la aptitud necesaria para lograr que tengan éxito en la vida.

En los últimos años se ha utilizado lo que se conoce como Inteligencia Emocional (IE) para aplicarlo en el desarrollo de las aptitudes emocionales en los estudiantes y así contribuir a una formación integral.

Comenzaremos por definir el objetivo nuestro trabajo, luego conoceremos las competencias genéricas en la EMS en México, para luego entender ¿qué es el aprendizaje y la inteligencia emocional? Se sigue con la propuesta de tips para el desarrollo de la Inteligencia Emocional y se concluye con algunas reflexiones para que los docentes tomen en consideración al aplicar estos tips en el aula.

\section{OBJETIVO}

Este trabajo basado en nuestra experiencia docente tiene como finalidad proponer tips que el docente puede utilizar en el aula, para hacer consiente al alumno de sus emociones y pueda desarrollar su Inteligencia Emocional y esta a su vez impacte en su proceso de aprendizaje ayudando al desarrollo de competencias. Dichos tips son para aplicar en la Educación Media Superior.

\subsection{COMPETENCIAS GENÉRICAS EN LA EDUCACIÓN MEDIA SUPERIOR EN MÉXICO}

El Sistema Nacional de Bachillerato establece las competencias que constituyen el marco curricular común de la EMS, las cuales se dividen en: Genéricas, Disciplinares y Profesionales.

Las competencias genéricas son competencias clave, por su importancia y aplicaciones diversas a lo largo de la vida; transversales, por ser relevantes a todas las disciplinas y espacios curriculares de la 
EMS, y transferibles, por reforzar la capacidad de los estudiantes de adquirir otras competencias (SEMS, 2008.) Estas competencias nos llevan a un desarrollo integral. En total son 11 competencias genéricas y cada una presenta ciertos atributos.

1. Se conoce y valora a sí mismo y aborda problemas y retos teniendo en cuenta los objetivos que persigue.

2. Es sensible al arte y participa en la apreciación e interpretación de sus expresiones en distintos géneros.

3. Elige y practica estilos de vida saludable.

4. Escucha, interpreta y emite mensajes pertinentes en distintos contextos mediante la utilización de medios, códigos y herramientas apropiados.

5. Desarrolla innovaciones y propone soluciones a problemas a partir de métodos establecidos.

6. Sustenta una postura personal sobre temas de interés y relevancia general, considerando otros puntos de vista de manera crítica y reflexiva.

7. Aprende por iniciativa e interés propio a lo largo de la vida.

8. Participa y colabora de manera efectiva en equipos diversos.

9. Participa con una conciencia cívica y ética en la vida de su comunidad, región, México y el mundo.

10. Mantiene una actitud respetuosa hacia la interculturalidad y la diversidad de creencias, valores, ideas y prácticas sociales.

11. Contribuye al desarrollo sustentable de manera crítica, con acciones responsables.

Dichas competencias se distribuyen a lo largo de los tres años de estudio del bachillerato (EMS) y se deben desarrollar en cada asignatura. Las circunstancias del mundo actual requieren que los jóvenes sean personas reflexivas, capaces de desarrollar opiniones, interactuar en contextos plurales, asumir un papel propositivo como miembros de la sociedad. Estas competencias asumen el reto de poder formar futuros hombre que sean conscientes de cuidar el mundo donde viven y poder relacionarse de manera armoniosa con la sociedad, sin descuidar el desarrollo científico.

Debemos entender que el alumno debe estar en la mejor disposición para que pueda adquirir una educación Integral, pero también depende de los docentes crear el ambiente propicio para fomentar el desarrollo de las competencias. Desde la primera competencia se puede ver que no solo se necesita la parte académica sino es de suma importancia el desarrollo emocional de los jóvenes. "No podemos seguir construyendo gente con mentes de altos alcances intelectuales, pero analfabetas emocionales" (Ladrón de Guevara, 2014, p.8) 


\subsection{APRENDIZAJE}

En el área de educación el alumno tiene que aprender; según en el informe de la UNESCO de la Comisión Internacional sobre la educación en el siglo XXI, se mencionan los cuatro pilares de la educación: aprender a conocer, aprender a hacer, aprender a vivir juntos y aprender a ser (UNESCO, 2015). Si la educación consiste en aprender, entonces ¿qué es el aprendizaje?; la mayoría de los autores coinciden en señalar que es un proceso donde se adquieren ciertos tipos de conocimientos. Una definición más específica es: “... proceso mediante el cual una persona adquiere destrezas o habilidades prácticas (motoras o intelectuales), incorpora contenidos informativos o adopta nuevas estrategias de conocimiento y/o acción" (Garza \& Leventhal, 2004, p. 48)

El hecho de adquirir significa que las personas ya tienen ciertas destrezas o habilidades naturales, innatas, así como también presentan cierto tipo de conocimiento; pero estos no son suficientes para poder subsistir en una sociedad que continuamente está evolucionando, por lo que es necesario el seguir aprendiendo. El aprendizaje depende de tener una buena actitud, para desarrollar una aptitud. Hay que saber diferenciar entre ambas, la actitud se define como la disposición de ánimo manifestada de algún modo y la aptitud es la capacidad para operar competentemente en una determinada actividad (RAE, 2017). La aptitud se puede adquirir a través del estudio y la constancia se puede ser apto para determinada actividad; la actitud es una conducta que puede ser modificada por las personas, podemos decir que es la manera de cómo enfrentar diferentes situaciones.

En el ámbito escolar y de manera general para poder aprender es necesario que las personas quieran y estén dispuestas a ello; influyen sus estados de ánimo, sus emociones y sus intereses particulares. Si el alumno está motivado en el estudio, le será más fácil comprender y retener el conocimiento; sino tiene interés en el estudio le será difícil poder aprender. Por lo tanto, se requiere que esté preparado emocionalmente. “... hay personas que son pensadores naturales... por sí mismos se enseñan a manejar las estructuras lógicas con gran afectividad, existen otras que... requieren de algún tipo de monitoreo para que puedan desarrollar habilidades que los preparen a aprender a aprender" (Garza \& Leventhal, 2004, p. 48).

Es aquí donde el docente juega un papel muy importante, su rol es guiar al alumno para que desarrolle sus habilidades tanto cognitivas como afectivas; conociendo ellos sus emociones, su manera de sentir y pensar, así como la manera de socializar, pueden entenderse mejor y apropiarse de los conocimientos eficazmente.

\subsection{INTELIGENCIA EMOCIONAL}

A partir de los años 90's se ha venido utilizando el termino inteligencia emocional como sinónimo de éxito en la vida. Los grandes estudiosos del tema indican que las emociones juegan un papel importante 
en el desarrollo de todo ser humano. Para Howard Gardner la IE es la forma de conectar las emociones con uno mismo; desde el punto de vista de Daniel Goleman, la inteligencia emocional implica la capacidad de una persona para desarrollar "habilidades tales como ser capaz de motivarse y persistir frente a las decepciones; controlar el impulso y demorar la gratificación, regular el humor y evitar que los trastornos disminuyan la capacidad de pensar; mostrar empatía y abrigar esperanzas” (Goleman, 2007, p. 54). Es la forma en que la persona interactúa con otras, donde entran en juego sus sentimientos sus actitudes, habilidades, destrezas... que lo ayudan a establecer una relación con el mundo.

En este mismo sentido y aplicando este concepto, dentro de las instituciones educativas, el alumno podrá dirigir, encauzar y aplicar sus conocimientos permitiendo que las emociones trabajen a favor de su personalidad, de esta forma se pueden guiar las actitudes de su vida hacia pensamientos y hábitos constructivos, lograr el éxito en todos los aspectos. A causa de las emociones, los pensamientos y las acciones se entrelazan y permiten un desarrollo positivo.

Gran parte del aprendizaje formal se obtiene a partir de las exposiciones en las aulas; es decir el maestro da la información necesaria de un tema en particular y cada alumno capta lo más importante para él; lo que llama su atención, lo que le agrada, por lo que siente curiosidad. Esto hace que los alumnos procesen diversa información y quizá el maestro se esmera en dar toda esta cantidad de datos, pero la otra parte es la aptitud que desarrollará el alumno para apropiarse del conocimiento y luego para proyectarlo socialmente hablando.

Nos ha tocado vivir en una era digital donde ahora también se aprende a través de los medios de comunicación, desde el ámbito educativo hay que estar atentos con este nuevo rol que tendrá el docente, interactuando en contextos virtuales, donde los alumnos desarrollan también habilidades para la vida.

La gran cantidad de datos que se les da para que el alumno adquiera cierta aptitud, la manera como lo aprende y la actitud con la que recibe la información nos llevan a replantear la forma en que enseñamos.

\subsection{TIPS PARA EL DESARROLLO DE LA INTELIGENCIA EMOCIONAL}

Es importante como docentes brindarles la información necesaria para que el alumno aprenda, pero también hay que motivarlos, ayudándolos a desarrollar sus aptitudes personales. ¿Cómo podemos ayudarlos?

Primero tenemos que conocer que la inteligencia emocional, la podemos trabajar en dos partes:

La aptitud personal (conocimiento y manejo de uno mismo).

- Autoconocimiento. Conocimiento de los propios estados internos, preferencias, recursos e intuiciones.

- Autorregulación. Manejo de los propios estados internos, impulsos y recursos. 
- Motivación. Tendencias emocionales que guían o faciliten la obtención de metas.

La aptitud social (conocimiento e influencia positiva en los demás)

- Empatía. Captación de sentimientos, necesidades e intereses ajenos.

- Habilidades sociales. Poder convivir con los demás de manera armónica e inducir en los demás la respuesta deseable. (Goleman, 2002, p. 45-46)

¿Cómo podemos aplicarlo en el aula? No es necesario crear un curso a parte, ni alargar nuestro programa de estudio dándoles más información. Podemos incluirlo en cualquier área de conocimiento y de manera cotidiana en nuestro salón de clases.

Para poder lograr que el alumno tenga una aptitud personal y social debemos hacer que:

\title{
Tip 1: Conozca sus emociones
}

Ser joven no es fácil, se pasa por una etapa donde el cuerpo está cambiando y con ellos nuestras emociones.

\begin{abstract}
Mientras no entendamos nuestra mente y emociones haremos cosas que nos destruyen y no podremos dejar de hacerlas... Existe una serie de sustancias químicas llamadas neurotransmisores y hormonas que se riegan en tu cuerpo y te hacen experimentar toda la gama de emociones (Albarrán Gutiérrez, 2010, p. 23).
\end{abstract}

No es fácil esta etapa de la vida, pero no se pueden detener el aprendizaje académico, como docentes comprendemos que algunas veces los alumnos cambian de humor constantemente y si aunado a esto hacemos rígidas nuestras clases y con poca convivencia, es lógico esperar que se aburran y no entiendan las explicaciones en el aula. Si hacemos que ellos primero se conozcan, descubran sus sentimientos, identifiquen que son las emociones y se den cuenta de su realidad, entonces los estamos preparando para que logren un buen desarrollo de competencias.

Para lograr que el alumno conozca cuáles son sus emociones, podemos apoyarnos con diferentes pruebas, como, por ejemplo.

\begin{tabular}{|l|l|l|l|}
\hline N & CONOCIÉNDOME & SÍ & NO \\
\hline 1 & Mis compañeros se burlan de mí & & \\
\hline 2 & Soy una persona emprendedora & & \\
\hline 3 & Me cuesta trabajo tener amigos & & \\
\hline 4 & Estoy triste muchas veces & & \\
\hline 5 & Soy listo & & \\
\hline 6 & Soy tímido & & \\
\hline 7 & Mi cara me disgusta & & \\
\hline 8 & Cuando sea mayor voy a ser una persona importante & & \\
\hline 9 & Me preocupo mucho cuando presento un examen & & \\
\hline 10 & Cuando algo va mal suele ser por culpa mía & & \\
\hline 11 & Creo problemas en mi familia & & \\
\hline 12 & Soy fuerte & & \\
\hline
\end{tabular}




\begin{tabular}{|l|l|l|l|}
\hline 13 & Tengo buenas ideas & & \\
\hline 14 & Soy importante en mi familia & & \\
\hline 15 & Quiero ganar siempre & & \\
\hline 16 & Tengo habilidad con las manos & & \\
\hline 17 & Cuando las cosas son difíciles las dejo sin hacer & & \\
\hline 18 & Hago muchas cosas malas & & \\
\hline 19 & Dibujo bien & & \\
\hline 20 & Soy bueno para la música & & \\
\hline 21 & Me porto mal en casa & & \\
\hline 22 & Soy lento al hacer mi trabajo en la escuela & & \\
\hline 23 & Soy un miembro importante del grupo & & \\
\hline 24 & Soy nervioso & & \\
\hline 25 & Tengo ojos bonitos & & \\
\hline 26 & Dentro de la clase puedo dar una buena impresión & & \\
\hline 27 & A mis amigos les gustan mis ideas & & \\
\hline 28 & Frecuentemente me meto en problemas & & \\
\hline 29 & Tengo suerte & & \\
\hline 30 & Me preocupo mucho por las cosas & & \\
\hline 31 & Me gusta ser como soy & & \\
\hline 32 & Me siento un poco rechazado/a & & \\
\hline 33 & Me gustaría ser diferente a como soy & & \\
\hline 34 & A menudo soy antipático/a con los demás & & \\
\hline
\end{tabular}

Este es parte del cuestionario Conociéndome. (Albarrán Gutiérrez, 2010, p. 25-26)

Estas pruebas se pueden aplicar durante las primeras clases y es totalmente personal y es un documento que ellos deben conservar, para su posterior valoración. Algunas reacciones que hemos observado son: seriedad, risas, molestia, pena, etc. se les pide que lo lleven a su casa y terminen de contestarlo en la tranquilidad de su hogar. Veras como cambia el ambiente en el aula al día siguiente; sobre todo si inicias la clase con una lista de emociones pegada en algún lugar visible.

Se puede empelar también un ejercicio llamado informe meteorológico personal (Ladrón de Guevara, 2014, p.60), en el cual el alumno debe elaborar un reporte meteorológico sobre su tiempo interior, con la finalidad de aprender que las emocines están en movimiento y que es importante reconocerlas.

Apoyandonos con un fondo musical pedirles :

- Tómate un momento y siéntate tranquilo.

- Respira y préstale atención a tu respiración...dentro...fuera...dentro...fuera

- Coloca tu mano en el corazón

- ¿Cómo te sientes hoy?

- ¿Qué te dice tu corazón sobre cómo te sientes?

Ahí adentro ¿Está nublado o el sol brilla?, ¿llovizna o llueve a mares?, ¿Hace frío, calor, o es una temperatura suave?

Al finalizar con tres lápices de colores pídele que haga un dibujo de su tiempo interior. 
Con este tipo de actividades estaremos desarrollando la competencia número 1: que se conozca y valore a sí mismo y pueda abordar problemas retos teniendo en cuenta los objetivos que persigue.

\section{Tip 2: Equilibre sus emociones}

Generalmente en la etapa de la adolescencia los jóvenes son impulsivos, sufren ataques de furia, tienen una ira desbordante, etc. Según Albarrán (2010), la ira brota por la forma de percibir las cosas: Se desea que el mundo sea perfecto, pero la perfección solo existe en la imaginación.

Nuestros alumnos tienen que aprenden la autorregulación de sus emociones. Una vez que ellos reconocen sus emociones podemos trabajar alguna actividad para lograr que piensen en un equilibrio de emociones.

Existen algunas actividades que podemos implementar en el aula y utilizar solo unos minutos de nuestra clase, podemos usar las que nos brinda el programa Contruye T, la llamada: ¿Qué tan fácil te frustras? La podemos obtener en: http://construyet.sep.gob.mx/lecciones/estudiantes/

El objetivo es: Reconocer cómo se manifiesta el estrés en nuestro cuerpo e identificar nuevas estrategias para aprender a manejarlo. Se puede desarrollar en el aula y solo se necesita una cartulina $u$ hoja de rotafolio y plumón.

Se forman equipos de 5 personas, se solicita que cada equipo identifique las partes de su cuerpo que resiente el estrés, tales como: dolores de cabeza, de estómago, tensión muscular, etc. Luego se solicita que reflexionen la importancia de aprender a manejar situaciones de estrés para evitar afectaciones que puedan dañar la calidad de vida. Posteriormente se pide por equipo escribir en el rotafolio las estrategias para manejar el estrés. Luego se realiza un listo resumido de las aportaciones realizadas. Se cierra con preguntas: ¿Cómo seré una mejor persona?, ¿cómo seremos una mejor comunidad? Con este tipo de actividades aprendemos la forma que se manifiesta el estrés y que no somos los únicos con esas manifestaciones, pero al mismo tiempo conocemos formas de controlarlo. 


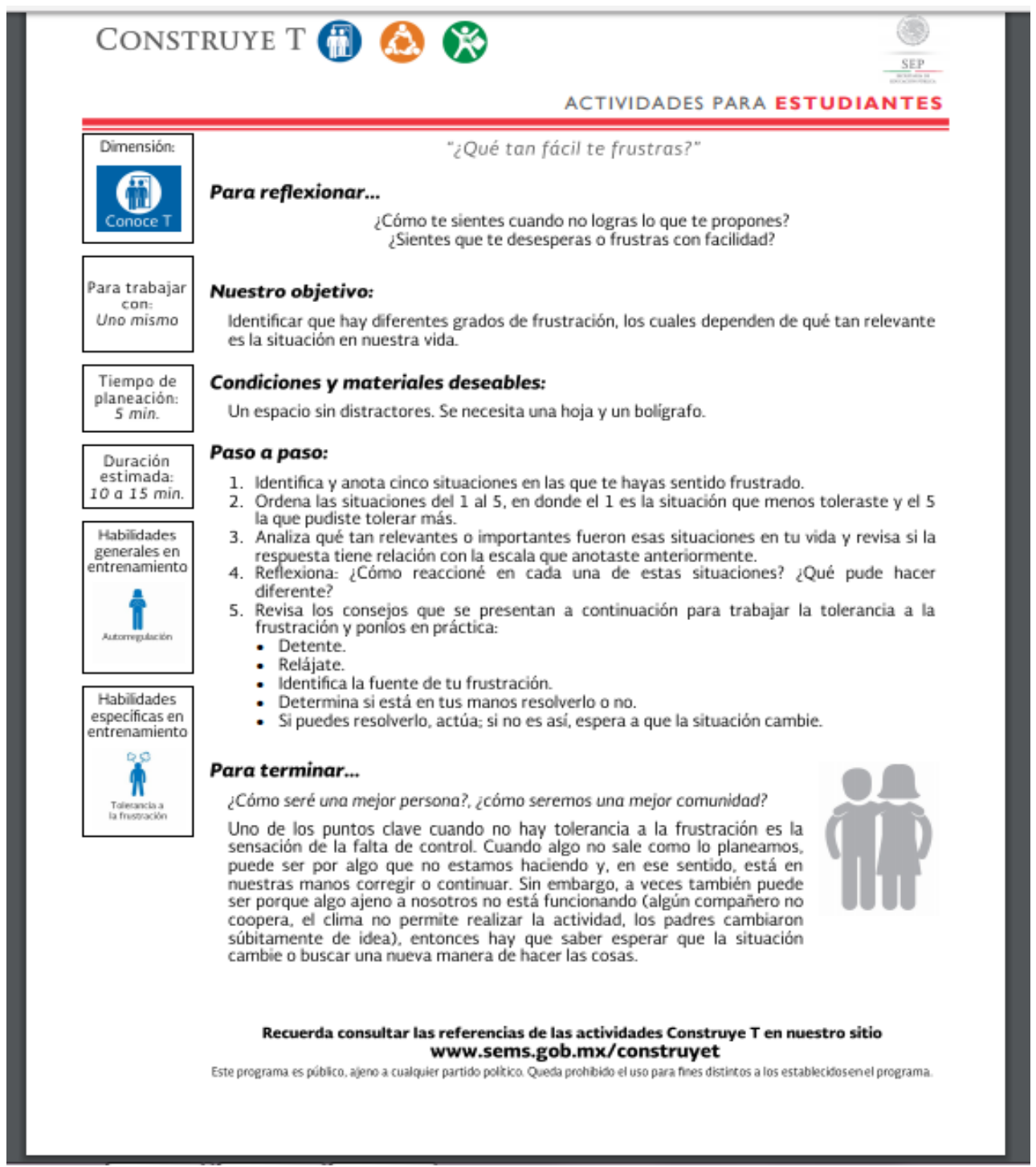

Estas fichas son muy fáciles de obtener y de aplicar en el aula. En ella encuentras las habilidades a desarrollar, invita a la reflexión con preguntas detonadoras, se indica el objetivo de la actividad, los pasos a seguir y cierra con una reflexión final. Desde luego que hay que saber elegirlas acorde al grupo con el que se está trabajando.

Equilibrando sus emociones puede desarrollar la competencia número 7: Él debe estar dispuesto a aprender por iniciativa e interés propio a lo largo de su vida.

\section{Tip 3: Tenga Automotivación}

Los jóvenes emocionalmente inteligentes son aquellos que siempre están motivados, tienen fuerza de voluntad para salir adelante ante cualquier situación. Para ello podemos sugerir a nuestros alumnos que lean libros de superación personal.

Algunos de ellos pueden ser: 
- El caballero de la armadura oxidada, de Robert Fisher

- El monje que vendió su Ferrari, de Robin Sharma

- El principito, de Antonie de Saint-Exupery

- Ami, el niño de las estrellas, de Enrique Barrios

- Etc.

A la hora de dar nuestra clase mencionamos frases de alguno de estos libros para despertar en ellos el interés de leer la obra completa.

De esta manera contribuye a la competencia 2: se hace sensible al arte y puede participar en la interpretación de expresiones de distintos géneros.

\section{Tip 4: Activación}

La activación física o cultural es parte importante en la formación de los jóvenes a través de estas actividades pueden convivir con otros compañeros y crear empatía. Pedro Albarrán (2010) maneja la siguiente lista de actividades las cuales se les puedes sugerir a los alumnos:

\begin{tabular}{|c|c|}
\hline $\begin{array}{l}\text { - } \text { Danza contemporánea } \\
\text { - Jazz } \\
\text { - } \text { Música (tocar un instrumento) } \\
\text { - Pintura en lienzo } \\
\text { - Escultura } \\
\text { - Deportes } \\
\text { - Ajedrez } \\
\text { - Buceo } \\
\text { - Creación literaria } \\
\text { - Teatro } \\
\text { - Campismo } \\
\text { - Yoga } \\
\text { - Tai chi } \\
\text { - Meditación } \\
\text { - Artes marciales } \\
\text { - Artesanías }\end{array}$ & $\begin{array}{l}\text { O se puede acudir a } \\
\text { - } \quad \text { Museos } \\
\text { - } \quad \text { Congresos } \\
\text { - Exposiciones } \\
\text { Por si fuera poco, } \\
\text { - Aprender a diseñar } \\
\text { videojuegos } \\
\text { - Escribir poemas } \\
\text { - Escribir un libro } \\
\text { - Aprender como iniciar un } \\
\text { negocio }\end{array}$ \\
\hline
\end{tabular}

Mientras los jóvenes realicen actividades que les hagan sentir bien, relajarse, utilizar la energía que poseen o realizar actividades gratificantes, estaremos logrando que tenga una buena disposición para el aprendizaje. Hay que dejar que desarrollen su creatividad. Así estaremos propiciando la competencia 3: elige y practica estilos de vida saludable. 


\title{
Tip 5: Trabajo en equipo
}

Una buena forma de ayudar a desarrollar la habilidad social y la empatía es precisamente el trabajo en equipo. Con él pueden desarrollar afecto, simpatía, comprensión, etc. creando mejores ambientes de estudio.

\begin{abstract}
Quienes hemos experimentado el trabajo en equipo sabemos que el hecho de compartir proyectos y metas es un poderoso motor motivacional que nos permite tomas riesgos y afrontar con mayor ahínco a las fuerzas que se oponen al cambio; de la misma manera, el trabajo se enriquece con la conjunción de diversas formas de pensar y de actuar propiciando la búsqueda constante de la innovación... (Frola \& Velásquez, 2011, p. 10)
\end{abstract}

Nuestras experiencias nos demuestran que día a día, nuestros alumnos no quieren trabajar en equipo, es decir físicamente. Las nuevas tecnologías de comunicación han cambiado la forma de trabajo colaborativo, esto no lo consideramos mal, al contrario, es algo que ayuda cuando no podemos asistir físicamente a las reuniones de trabajo y, sin embargo, podemos colaborar desde cualquier parte, solo se necesita estar conectados a Internet para compartir nuestras ideas y poder desarrollarlas. Pero es necesario, si se tiene la posibilidad, de que los jóvenes convivan físicamente y compartan la experiencia de trabajar coordinadamente durante la clase. Esto hace que se conozcan más entre ellos, adquiriendo una mayor confianza y seguridad al convivir con diferentes compañeros, que logren unificar criterios y juntos logren una meta.

La competencia 9 menciona que el alumno debe participar y colaborar de manera efectiva en equipos diversos, con ello puede aportar puntos de vista con apertura y considerar los de otras personas de manera reflexiva, asimiento una actitud constructiva, congruente con los conocimientos y habilidades con los que cuenta dentro de los distintos equipos de trabajo.

\section{Tips generales:}

Desde el inicio de nuestro trabajo aclaramos que estos tips los damos con base a nuestra experiencia personal, no somos expertas en la materia, sin embargo, lo compartimos con la finalidad de poder ayudar y hacer de la docencia una grata experiencia, sobre todo cuando trabajamos en las aulas. De manera general podemos hacer las siguientes sugerencias.

Como maestros tenemos que:

- $\quad$ crearles conciencia de que nadie es perfecto

- fijarle metas a corto plazo en cuanto al ámbito académico

- realzar las acciones positivas que realicen

- $\quad$ ser flexibles con ellos

- $\quad$ inducir la creatividad

- fomentar la actitud mental positiva 
- $\quad$ hacer que descubran sus limitaciones

- $\quad$ aplicar el uso de valores como: responsabilidad, honestidad, respecto, justicia ...

- establecer una buena comunicación alumno-maestro

Debemos tratar de que el alumno:

- desarrolle sus potencialidades humanas

- $\quad$ se entusiasme con los proyectos académicos

- $\quad$ trabaje proyectos para ser emprendedor

- $\quad$ se proponga retos

- desarrolle su proyecto de vida

- respete las opiniones de cada uno

- acepte a los demás tal como son

- aprenda a escuchar mientras se expone

- $\quad$ tenga sensibilidad hacia lo que otros dicen

- Trabaje de manera colaborativa en equipos

- $\quad$ ayude a sus compañeros

- dar y aceptar críticas constructivas

De esta manera le creamos una conciencia emocional y podemos felicitarlo u observar sus desaciertos. Debemos elegir las palabras y expresiones para poder incidir en su autoestima. Él se sentirá más seguro y quizá para el próximo tema podrá emitir sin miedo sus opiniones. El tema para tratar (información) podrá procesarla de manera más consiente apropiándose del conocimiento (aprende) y en algún momento de su vida podrá recrearlo.

Cuando se trabaja en el salón de clases el alumno recibe información por diversos medios, cada uno capta la información de diferente manera según su interés, es por eso que el maestro debe planear cuidadosamente las sesiones de clase para que favorezcan la percepción, atención, la motivación, el procesamiento de la información, la memoria y el aprendizaje. "La educación no consiste en rellenar la mente de los demás con ideas ajenas, sino en estimularla para que produzca sus propias ideas, ayudándolos a creer que pueden y podrán" (Ladrón de Guevara, 2014, p. 50)

De nada nos sirve darles día a día información, si él no se siente seguro, sino logra expresar o emitir una opinión en el salón de clases. Nos interesa que el alumno procese la información pero que también colabore y pueda ser capaz de emitir un juicio acerca del tema, que pueda convivir en armonía 
con sus compañeros, que pueda admitir una equivocación y rectificar su error, apoyar a sus compañeros de manera personal como académicamente.

\section{CONCLUSIONES}

El mundo se ha dado cuenta que es necesario educar emocionalmente a los jóvenes para que logren ser personas satisfechas y que generen el cambio en la humanidad. Es importante que ellos tomen conciencia de sus emociones para poder ayudar a mejorar las condiciones de vida actual. Se debe respetar las diferencias que cada uno siente con respecto a determinadas cosas.

Hoy en día nos sorprendemos de mirar que la tecnología avanza rápidamente y nosotros estamos inmersos en ella, tenemos información prácticamente a la mano, no hay excusas para no investigar o estudiar; sin embargo, también nos sorprende como es que jóvenes puedan cometer actos de barbarie y atentar contra la vida de otras personas; y nos impacta más que dichos jóvenes han destacado académicamente como buenos estudiantes, provienen de familias económicamente estables y sin antecedentes de mal comportamiento.

Todo esto nos hace pensar en que el esfuerzo que hacemos como docentes para preparar a nuestros alumnos, no es el suficiente y quizá la falla este precisamente en que no tomamos en cuenta el lado humano de estos jóvenes, quizá solo nos limitamos a darles la información y creemos que a través de los exámenes podemos valorar si aprendieron o no. Pero aprendieron ¿a qué? a memorizar la información dada, para después olvidarla cuando pasen al próximo curso escolar; realmente eso queremos o nos interese más el hecho que los jóvenes puedan estar motivados, que amen la vida que la disfruten que cada día de clases sea para ellos un nuevo reto, en el cual estén involucrados los conocimientos que les da el maestro, el trabajo en equipo, el respeto hacia los demás, el poder proyectar de alguna manera lo aprendido a su vida personal, familiar o escolar. Aprendiendo a utilizar pequeños tips para el desarrollo de la IE estamos haciéndoles la vida más fácil tanto a ellos como a nosotros mismos, al mismo tiempo estamos desarrollando las competencias genéricas que indica nuestro modelo educativo, estamos haciendo que aprendan a aprender.

La IE nos permite como docentes preparar a los alumnos emocionalmente para que ellos puedan adoptar la mejor manera de aprender la información que se les da en el salón de clases y puedan desarrollar las competencias genéricas requeridas en el nivel medio superior, pero al mismo tiempo los preparamos para la vida misma.

El desarrollo de la aptitud personal y social nos permitirá trabajar armoniosamente en el aula e incidir en un aprendizaje significativo para su formación integral. Lo importante es que nuestros alumnos 
descubran el modo más fácil de desempeñarse para encauzar su vida y sus aspiraciones, el equilibrio de las emociones conduce a la armonía, satisfacción y al éxito.

Si como docente educamos las emociones, no vamos a evaluar con un examen final para aprobar o reprobar las emociones de nuestros alumnos, es la misma vida la que pone la prueba final. 


\section{REFERENCIAS}

Albarrán Gutierrez, P. E. (2010). Inteligrnacia Emocional para jóvenes (1a ed.). New Delhi, India: EMU Editores Mexicanos Unidos, Colección Diamante.

Fernández-Berrocal, P. \& Ruiz Aranda D. (2008). La Inteligencia Emocional en la Educación. Revista electrónica de Investigación Psicoeducativa, 6(2), pp. 421-436.

Frola, P. \& Velásquez, J. (2011). Creatividad en los equipos. (1a ed.) México: Centro de Investigación Educativa y Capacitación Institucional.

Garza, R. M., \& Leventhal, S. (2004). Aprender cómo aprender (3a ed.). México: Trillas.

Goleman, D. (2002). La inteligencia emocional en la empresa (3a ed.). España. Editorial Vergara.

Goleman, D. (2007). La inteligencia emocional (44a reimpresión). México: Javier Vergara Editor.

Jackes Delors J. (1996). La Educación encierra un tesoro. Madrid, España: Santillana. ediciones UNESCO.

Ladrón de Guevara, L. (2014). Reingeniería del éxito educativo. México: Frovel Editorial.

RAE (2017). Diccionario de la Real Academia Española. Recuperado de https://dle.rae.es/actitud?m=form

Secretaria de Educación Pública SEP (2015) Programa ConstuyeT. Recuperado de http://www.gob.mx/sep/acciones-y-programas/programa-construye-t-4598

Secretaria de Educación Media Superior SEMS (2008). Acuerdos Secretariales. Acuerdo 444. Recuperado de http://www.sems.gob.mx/en_mx/sems/acuerdo_secretarial 